\title{
Secure Implementation in Queueing Problems
}

\author{
Katsuhiko Nishizaki \\ Graduate School of Economics, Osaka University, Toyonaka, Japan \\ Email: gge008nk@mail2.econ.osaka-u.ac.jp
}

Received August 3, 2012; revised September 5, 2012; accepted October 7, 2012

\begin{abstract}
This paper studies secure implementability (T. Saijo, T. Sjöström and T. Yamato, "Secure Implementation," Theoretical Economics, Vol. 2, No. 3, 2007, pp. 203-229) in queueing problems. Our main result shows that the social choice function satisfies strategy-proofness and strong non-bossiness (Z. Ritz, "Restricted Domains, Arrow-Social Welfare Functions and Noncorruptible and Non-Manipulable Social Choice Correspondences: The Case of Private Alternatives," Mathematical Social Science, Vol. 4, No. 2, 1983, pp. 155-179), both of which are necessary for secure implementation, if and only if it is constant on the domains that satisfy weak indifference introduced in this paper. Weak indifference is weaker than minimal richness (Y. Fujinaka and T. Wakayama, "Secure Implementation in Economies with Indivisible Objects and Money," Economics Letters, Vol. 100, No. 1, 2008, pp. 91-95). Our main result illustrates that secure implementation is too difficult in queueing problems since many reasonable domains satisfy weak indifference, for example, convex domains.
\end{abstract}

Keywords: Secure Implementation; Dominant Strategy Implementation; Nash Implementation; Strategy-Proofness; Queueing Problems

\section{Introduction}

In this paper, we consider queueing problems of allocating positions in a queue to agents, each of whom has a constant unit waiting cost, with monetary transfers. Examples of such problems are the use of large-scaled experimental installations, event sites, and so forth ${ }^{1}$.

Strategy-proofness is a standard property for nonmanipulability: The truthful revelation is a weakly dominant strategy for each agent. However, the strategyproof mechanism might have a Nash equilibrium which induces a non-optimal outcome. This problem is solved by secure implementation (Saijo, et al. [2]), that is, double implementation in dominant strategy equilibria and Nash equilibria ${ }^{2}$. Previous studies illustrate how difficult it is to find desirable and securely implementable social choice functions: Voting environments (Saijo, et al. [2]; Berga and Moreno [4]), public good economies (Saijo, et al. [2]; Nishizaki [5]), pure exchange economies (Mizukami and Wakayama [6]; Nishizaki [7]), the problems of providing a divisible and private good with monetary transfers (Saijo, et al. [2]; Kumar [8]), the problems of allocating indivisible and private goods with monetary transfers (Fujinaka and Wakayama [9]), Shapley-Scarf housing markets (Fujinaka and Waka-

\footnotetext{
${ }^{1}$ For queueing problems, see Suijs [1] and so forth.

${ }^{2}$ This concept is considered to be a benchmark for constructing mechanisms which work well in laboratories. See Cason, et al. [3] for experimental results.
}

yama [10]), and allotment economies with single-peaked preferences (Bochet and Sakai [11]).

This paper is most closely related to the one written by Fujinaka and Wakayama [9]. They show a constancy result on secure implementation when the domain satisfies minimal richness (Fujinaka and Wakayama [9]). Our model is a special case of their one and have many reasonable domains which do not satisfy minimal richness. On the basis of this fact, we study the possibility of secure implementation in queueing problems. Unfortunately, our main result shows that only constant social choice functions satisfy strategy-proofness and strong non-bossiness (Ritz [12]), both of which are necessary for secure implementation, on the domains satisfy weak indifference, which is weaker than minimal richness, introduced in this paper.

This paper is organized according to the following sections. In Section 2, we introduce our model, properties of social choice functions, and domain-richness conditions. We show our results in Section 3. Section 4 concludes this paper.

\section{Notation and Definitions}

Let $I \equiv\{1, \cdots, n\}(n \geq 2)$ be a set of agents. Let $\sigma \equiv\left(\sigma_{i}\right)_{i \in I} \in I^{n}$ be a queue, where, for each $i \in I, \sigma_{i}$ is the position for agent $i$ in the queue $\sigma$ and for each $i, j \in I$ with $i \neq j, \sigma_{i} \neq \sigma_{j}$. For each $i \in I$, let $\left(\sigma_{i}, t_{i}\right) \in I \times \mathbb{R}$ be a consumption bundle for agent $i$, 
where $t_{i}$ is a monetary transfer for agent $\boldsymbol{i}$. Let $t \equiv\left(t_{i}\right)_{i \in I} \in \mathbb{R}^{n}$ be a profile of monetary transfers and $(\sigma, t) \in I^{n} \times \mathbb{R}^{n}$ be a profile of consumption bundles, called an allocation. Let

$$
Z \equiv\left\{(\sigma, t) \in I^{n} \times \mathbb{R}^{n} \mid \sigma \text { is a queue and } \sum_{k \in I} t_{k} \leq 0\right\}
$$

be the set of feasible allocations.

For each $i \in I$, let $c_{i} \in \mathbb{R}_{++} \equiv\{x \in \mathbb{R} \mid x>0\}$ be a unit waiting cost for agent $i$ and $C_{i} \subseteq \mathbb{R}_{++}$be a set of unit waiting costs for agent $i$. For each $i \in I$, let $u: I \times \mathbb{R} \times C_{i} \rightarrow \mathbb{R}$ be the utility function for agent $i$ such that for each $\left(\sigma_{i}, t_{i}\right) \in I \times \mathbb{R}$ and each $c_{i} \in C_{i}$,

$$
u\left(\sigma_{i}, t_{i} ; c_{i}\right) \equiv-\left(\sigma_{i}-1\right) c_{i}+t_{i} .
$$

Let $C \equiv \prod_{i \in I} C_{i}$ be the domain and $c \equiv\left(c_{i}\right)_{i \in I} \in C$ be a profile of unit waiting costs. For each $i \in I$, let $c_{-i} \equiv\left(c_{j}\right)_{j \neq i} \in C_{-i} \equiv \prod_{j \neq i} C_{j}$ be a profile of unit waiting costs for agents other than agent $i$.

Let $f: C \rightarrow Z$ be a social choice function. For each $c \in C$, let $(\sigma(c), t(c)) \in Z$ be the allocation associated with the social choice function $f$ at the profile of unit waiting costs $c$ and $\left(\sigma_{i}(c), t_{i}(c)\right) \in I \times \mathbb{R}$ be the consumption bundle for agent $i \in I$ in the allocation $(\sigma(c), t(c))$.

Saijo et al. [2] show that strategy-proofness and strong non-bossiness are necessary for secure implementation.

Definition 1 The social choice function $f$ satisfies strategy-proofness if and only if for each $c, c^{\prime} \in C$ and each $i \in I$,

$$
\begin{aligned}
& -\left(\sigma_{i}\left(c_{i}, c_{-i}^{\prime}\right)-1\right) c_{i}+t_{i}\left(c_{i}, c_{-i}^{\prime}\right) \\
& \geq-\left(\left(\sigma_{i}\left(c_{i}^{\prime}, c_{-i}^{\prime}\right)-1\right) c_{i}\right)+t_{i}\left(c_{i}^{\prime}, c_{-i}^{\prime}\right)
\end{aligned}
$$

Definition 2 The social choice function $f$ satisfies strong non-bossiness if and only if for each $c, c^{\prime} \in C$ and each $i \in I$, if

$$
\begin{gathered}
-\left(\sigma_{i}\left(c_{i}, c_{-i}\right)-1\right) c_{i}+t_{i}\left(c_{i}, c_{-i}\right) \\
=-\left(\sigma_{i}\left(c_{i}^{\prime}, c_{-i}\right)-1\right) c_{i}+t_{i}\left(c_{i}^{\prime}, c_{-i}\right) \\
\text { then }\left(\sigma\left(c_{i}, c_{-i}\right), t\left(c_{i}, c_{-i}\right)\right)=\left(\sigma\left(c_{i}^{\prime}, c_{-i}\right), t\left(c_{i}^{\prime}, c_{-i}\right)\right)
\end{gathered}
$$

Fujinaka and Wakayama [9] show a constancy result on secure implementation when the domain satisfies minimal richness.

Definition 3 The domain $C$ satisfies minimal richness if and only if for each $i \in I$, each $c_{i}^{\prime}, c_{i}^{\prime \prime} \in C_{i}$, each $\sigma_{i}^{\prime}, \sigma_{i}^{\prime \prime} \in I$, and each $T \in \mathbb{R}$, if

$\left(\sigma_{i}^{\prime \prime}-\sigma_{i}^{\prime}\right) \sigma_{i}^{\prime \prime}<T<\left(\sigma_{i}^{\prime \prime}-\sigma_{i}^{\prime}\right) c_{i}^{\prime}$, then there exists $c_{i} \in C_{i}$ such that

1) $\left(\sigma_{i}^{\prime \prime}-\sigma_{i}^{\prime}\right) c_{i}=T$ and

2) $\left(\sigma_{i}^{\prime \prime}-\sigma_{i}\right) c_{i} \leq\left(\sigma_{i}^{\prime \prime}-\sigma_{i}\right) c_{i}^{\prime}$ for each $\sigma_{i} \in I \backslash\left\{\sigma_{i}^{\prime}, \sigma_{i}^{\prime \prime}\right\}$.

The following example shows that many reasonable domains do not satisfy minimal richness in our model.
Example 1 Let $i \in I$ and $C_{i}=\mathbb{R}_{++}$. Moreover, let $c_{i}^{\prime}=3, c_{i}^{\prime \prime}=1, \sigma_{i}^{\prime}=1, \sigma_{i}^{\prime \prime}=2$ and $T=2$. In this case, we have $\left(\sigma_{i}^{\prime \prime}-\sigma_{i}^{\prime}\right) c_{i}^{\prime \prime}=1<T<3=\left(\sigma_{i}^{\prime \prime}-\sigma_{i}^{\prime}\right) c_{i}^{\prime}$. Let $c_{i} \in C_{i}$ be such that $\left(\sigma_{i}^{\prime \prime}-\sigma_{i}^{\prime}\right) c_{i}=T$, that is, $c_{i}=2$. This implies that condition 1) in Definition 3 holds. On the other hand, if $c_{i}=2$, then

$\left(\sigma_{i}^{\prime \prime}-\sigma_{i}\right) c_{i}=-2>-3=\left(\sigma_{i}^{\prime \prime}-\sigma_{i}\right) c_{i}^{\prime}$ for $\sigma_{i}=3$. This implies that condition 2) in Definition 3 does not hold.

Our main result implies a constancy result on secure implementation when the domain satisfies weak indifference which is weaker than minimal richness.

Definition 4 The domain $C$ satisfies weak indifference if and only if for each $i \in I$, each $c_{i}^{\prime}, c_{i}^{\prime \prime} \in C_{i}$, each $\sigma_{i}^{\prime}, \sigma_{i}^{\prime \prime} \in I$, and each $T \in \mathbb{R}$, if

$\left(\sigma_{i}^{\prime \prime}-\sigma_{i}^{\prime}\right) c_{i}^{\prime \prime}<T<\left(\sigma_{i}^{\prime \prime}-\sigma_{i}^{\prime}\right) c_{i}^{\prime}$, then there exists $c_{i} \in C_{i}$ such that

$$
\left(\sigma_{i}^{\prime \prime}-\sigma_{i}^{\prime}\right) c_{i}=T \text {. }
$$

Remark 1 In our model, weak indifference is equivalent to convexity ${ }^{3}$.

\section{Results}

For simplicity of notation, let $\sigma_{i} \equiv \sigma_{i}\left(c_{i}, c_{-i}\right)$, $\sigma_{i}^{\prime} \equiv \sigma_{i}\left(c_{i}^{\prime}, c_{-i}\right), \quad \sigma_{i}^{\prime \prime} \equiv \sigma_{i}\left(c_{i}^{\prime \prime}, c_{-i}\right), \quad \sigma_{i}^{*} \equiv \sigma_{i}\left(c_{i}^{*}, c_{-i}\right)$, $\sigma_{i}^{* *} \equiv \sigma_{i}\left(c_{i}^{* *}, c_{-i}\right) \quad$ and $t_{i} \equiv t_{i}\left(c_{i}, c_{-i}\right), \quad t_{i}^{\prime} \equiv t_{i}\left(c_{i}^{\prime}, c_{-i}\right)$, $t_{i}^{\prime \prime} \equiv t_{i}\left(c_{i}^{\prime \prime}, c_{-i}\right), \quad t_{i}^{*} \equiv t_{i}\left(c_{i}^{*}, c_{-i}\right), \quad t_{i}^{* *} \equiv t_{i}\left(c_{i}^{* *}, c_{-i}\right) \quad$ for each $c, c^{\prime} \in C$ and each $i \in I$.

\subsection{Preliminary Results}

In this subsection, we assume that the social choice function $f$ satisfies strategy-proofness.

Lemma 1 shows that each agent's monetary transfer depends on her position in the queue given unit waiting costs for other agents. Since the proof is similar to Fujinaka and Wakayama [9], it is omitted.

Lemma 1 For each $c, c^{\prime} \in C$ and each $i \in I$, if $\sigma_{i}=\sigma_{i}^{\prime}$, then $t_{i}=t_{i}^{\prime}$.

Lemma 2 shows that if there exists a unit waiting cost such that some two different consumption bundles are indifferent in terms of utility level, then the position associated with the unit waiting cost is in between the two positions. In Lemma 2, we use the following notation: for each $i \in I$, each $c_{i} \in C_{i}$, each $\left(\sigma_{i}, t_{i}\right) \in I \times \mathbb{R}$, and each $\sigma_{i}^{\prime} \in I$, let $\boldsymbol{t}_{\boldsymbol{i}}\left(\sigma_{i}^{\prime} ;\left(\sigma_{i}, t_{i}\right), c_{i}\right) \equiv\left(\sigma_{i}^{\prime}-\sigma_{i}\right) c_{i}+t_{i}$.

Lemma 2 For each $c, c^{\prime} \in C$ and each $i \in I$, if $\sigma_{i}<\sigma_{i}^{\prime}$ and there exists $c_{i}^{\prime \prime} \in C_{i}$ such that

$-\left(\sigma_{i}-1\right) c_{i}^{\prime \prime}+t_{i}=-\left(\sigma_{i}^{\prime}-1\right) c_{i}^{\prime \prime}+t_{i}^{\prime}$, then $\sigma_{i} \leq \sigma_{i}^{\prime \prime} \leq \sigma_{i}^{\prime}$.

Proof. Suppose, by contradiction, that there exist $c, c^{\prime} \in C$ and $i \in I$ such that $\sigma_{i}<\sigma_{i}^{\prime}$,

$-\left(\sigma_{i}-1\right) c_{i}^{\prime \prime}+t_{i}=-\left(\sigma_{i}^{\prime}-1\right) c_{i}^{\prime \prime}+t_{i}^{\prime}$ for some $c_{i}^{\prime \prime} \in C_{i}$, and

${ }^{3}$ For the relationship among weak indifference and certain domainrichness conditions, see Appendix in Nishizaki [13]. 
$\sigma_{i}^{\prime \prime}<\sigma_{i}$ or $\sigma_{i}^{\prime}<\sigma_{i}^{\prime \prime}$. We consider the case of $\sigma_{i}^{\prime \prime}<\sigma_{i}$. By the hypothesis, we have

$$
c_{i}^{\prime \prime}=\frac{t_{i}^{\prime}-t_{i}}{\sigma_{i}^{\prime}-\sigma_{i}} .
$$

By the definition of $\boldsymbol{t}_{\boldsymbol{i}}$, we have

$$
c_{i}=\frac{\boldsymbol{t}_{\boldsymbol{i}}\left(\sigma_{i}^{\prime} ;\left(\sigma_{i}, t_{i}\right), c_{i}\right)-t_{i}}{\sigma_{i}^{\prime}-\sigma_{i}} .
$$

By the definition of $\boldsymbol{t}_{\boldsymbol{i}}$ and strategy-proofness, we have

$$
t_{i}^{\prime}<\boldsymbol{t}_{i}\left(\sigma_{i}^{\prime} ;\left(\sigma_{i}, t_{i}\right), c_{i}\right) \cdot{ }^{4}
$$

By Equations (1)-(3) and $\sigma_{i}<\sigma_{i}^{\prime}$, we have $c_{i}^{\prime \prime}<c_{i}$. Since we consider the case of $\sigma_{i}^{\prime \prime}<\sigma_{i}$, this implies

$$
\boldsymbol{t}_{\boldsymbol{i}}\left(\sigma_{i}^{\prime \prime} ;\left(\sigma_{i}, t_{i}\right), c_{i}\right)<\boldsymbol{t}_{\boldsymbol{i}}\left(\sigma_{i}^{\prime \prime} ;\left(\sigma_{i}, t_{i}\right), c_{i}^{\prime \prime}\right) \text {. }
$$

By the definition of $\boldsymbol{t}_{\boldsymbol{i}}$ and strategy-proofness, we have $\boldsymbol{t}_{\boldsymbol{i}}\left(\sigma_{i}^{\prime \prime} ;\left(\sigma_{i}, t_{i}\right), c_{i}^{\prime \prime}\right) \leq t_{i}^{\prime \prime} \leq \boldsymbol{t}_{\boldsymbol{i}}\left(\sigma_{i}^{\prime \prime} ;\left(\sigma_{i}, t_{i}\right), c_{i}\right)$. This is a contradiction to Equation (4). Similarly, we have a contradiction to strategy-proofness in the case of $\sigma_{i}^{\prime}<\sigma_{i}^{\prime \prime}$.

\subsection{Main Result}

Theorem 1 Suppose that the domain $C$ satisfies weak indifference. The social choice function $f$ satisfies strategy-proofness and strong non-bossiness if and only if it is constant ${ }^{5}$.

Proof. Since the "if" part is obvious, we only prove the "only if" part. Let $c, c^{\prime} \in C$. Firstly, we show $\left(\sigma\left(c_{i}, c_{-i}\right), t\left(c_{i}, c_{-i}\right)\right)=\left(\sigma\left(c_{i}^{\prime}, c_{-i}\right), t\left(c_{i}^{\prime}, c_{-i}\right)\right)$ for each $i \in I$. Suppose, by contradiction, that there exists $j \in I$ such that

$\left(\sigma\left(c_{j}, c_{-j}\right), t\left(c_{j}, c_{-j}\right)\right) \neq\left(\sigma\left(c_{j}^{\prime}, c_{-j}\right), t\left(c_{j}^{\prime}, c_{-j}\right)\right)$. By

strong non-bossiness and strategy-proofness, this implies

$$
-\left(\sigma_{j}-1\right) c_{j}+t_{j}>-\left(\sigma_{j}^{\prime}-1\right) c_{j}+t_{j}^{\prime} .
$$

By Lemma 1, this implies $\sigma_{j}<\sigma_{j}^{\prime}$ or $\sigma_{j}>\sigma_{j}^{\prime}$. Since $\left(\sigma\left(c_{j}, c_{-j}\right), t\left(c_{j}, c_{-j}\right)\right) \neq\left(\sigma\left(c_{j}^{\prime}, c_{-j}\right), t\left(c_{j}^{\prime}, c_{-j}\right)\right)^{j}$, by strong non-bossiness and strategy-proofness, it also implies

$$
-\left(\sigma_{j}-1\right) c_{j}^{\prime}+t_{j}<-\left(\sigma_{j}^{\prime}-1\right) c_{j}^{\prime}+t_{j}^{\prime}
$$

By Equations (5) and (6), we have $\left(\sigma_{j}^{\prime}-\sigma_{j}\right) c_{j}^{\prime}<t_{j}^{\prime}-t_{j}<\left(\sigma_{j}^{\prime}-\sigma_{j}\right) c_{j}$. Since $C$ satisfies weak indifference, this implies that there exists $c_{j}^{\prime \prime} \in C_{j}$ such that

\footnotetext{
${ }^{4}$ Note that the equality does not hold. If it holds, then we have $c_{i}=c_{i}^{\prime \prime}$ by Equations (1) and (2). This implies that $f$ should be a correspondence since we consider the case of $\sigma_{i}^{\prime \prime}<\sigma_{i}$.

${ }^{5}$ For the tightness of this theorem, see Examples 2, 3, and 4 in Nishizaki [13].
}

$$
-\left(\sigma_{j}-1\right) c_{j}^{\prime \prime}+t_{j}=-\left(\sigma_{j}^{\prime}-1\right) c_{j}^{\prime \prime}+t_{j}^{\prime} .
$$

We consider the case of $\sigma_{j}<\sigma_{j}^{\prime}$. In this case, by Lemma 2, we have $\sigma_{j} \leq \sigma_{j}^{\prime \prime} \leq \sigma_{j}^{\prime}$. If $\sigma_{j}^{\prime \prime}=\sigma_{j}$ or $\sigma_{j}^{\prime \prime}=\sigma_{j}^{\prime}$, then, by Equation (7) and strong nonbossiness, we have $\sigma_{j}=\sigma_{j}^{\prime}$. This is a contradiction. Therefore, we know

$$
\sigma_{j}<\sigma_{j}^{\prime \prime}<\sigma_{j}^{\prime}
$$

By applying the above argument to the left inequality repeatedly, we can find $c_{j}^{*}, c_{j}^{* *} \in C_{j}$ such that $\sigma_{j}<\sigma_{j}^{*}$ and $-\left(\sigma_{j}-1\right) c_{j}^{* *}+t_{j}=-\left(\sigma_{j}^{*}-1\right) c_{j}^{* *}+t_{j}^{*}$, where there exists no position between $\sigma_{j}$ and $\sigma_{j}^{*}$ induced by a unit waiting cost for agent $i$ given $c_{-j}$. In this case, we have $\sigma_{j}^{* *}=\sigma_{j}$ or $\sigma_{j}^{* *}=\sigma_{j}^{*}$. By strong non-bossiness, these imply $\sigma_{j}=\sigma_{j}^{*}$. This is a contradiction. Similarly, we have a contradiction in the case of $\sigma_{j}>\sigma_{j}^{\prime}$.

Without loss of generality, let $i=1$. Therefore, we have

$$
\left(\sigma\left(c_{1}, c_{-1}\right), t\left(c_{1}, c_{-1}\right)\right)=\left(\sigma\left(c_{1}^{\prime}, c_{-1}\right), t\left(c_{1}^{\prime}, c_{-1}\right)\right) .
$$

By the same argument stated above, we also have

$$
\begin{aligned}
& \left(\sigma\left(c_{1}^{\prime}, c_{2}, c_{-1,2}\right), t\left(c_{1}^{\prime}, c_{2}, c_{-1,2}\right)\right) \\
& =\left(\sigma\left(c_{1}^{\prime}, c_{2}^{\prime}, c_{-1,2}\right), t\left(c_{1}^{\prime}, c_{2}^{\prime}, c_{-1,2}\right)\right),
\end{aligned}
$$

where $C_{-1,2}$ is a profile of unit waiting costs for agents other than agents 1 and 2. By Equations (8) and (9), we have

$$
\begin{aligned}
& \left(\sigma\left(c_{1}, c_{2}, c_{-1,2}\right), t\left(c_{1}, c_{2}, c_{-1,2}\right)\right) \\
& =\left(\sigma\left(c_{1}^{\prime}, c_{2}^{\prime}, c_{-1,2}\right), t\left(c_{1}^{\prime}, c_{2}^{\prime}, c_{-1,2}\right)\right)
\end{aligned} .
$$

By sequentially replacing $c_{j}$ by $c_{j}^{\prime}$ for each $j \neq 1,2$ in this manner, we finally prove

$(\sigma(c), t(c))=\left(\sigma\left(c^{\prime}\right), t\left(c^{\prime}\right)\right)$.

Remark 2 The above theorem does not depend on the finiteness of the number of positions, which is used to prove Claim 3 in Proposition 1 of Fujinaka and Wakayama [9].

Obviously, constant social choice functions are securely implementable. Therefore, by bringing the above theorem together with a characterization of securely implementable social choice functions by Saijo et al. [2], we have the following constancy result on secure implementation.

Corollary 1 Suppose that the domain satisfies weak indifference. The social choice function is securely implementable if and only if it is constant.

Remark 3 In our model, Maskin monotonicity is not stronger than strategy-proofness ${ }^{6}$. This relationship implies that our main result is established by secure im-

${ }^{6}$ For this relationship, see Remark 6 in Nishizaki [13]. 
plementability but not by Nash implementability.

Remark 4 Saijo [14] shows the following constancy result on "Nash" implementation: The social choice function satisfies Maskin monotonicity and dual dominance (Saijo [14]) if and only if it satisfies constancy. In line with such domination, Fujinaka and Wakayama [9] show the following constancy result on "secure" implementation: The securely implementable social choice function satisfies non-dominance (Fujinaka and Wakayama [9]) if and only if it satisfies constancy. Note that nondominance is weaker than dual dominance ${ }^{7}$. In our model, similar to the relationship between minimal richness and weak indifference, we have a constancy result on secure implementation by a weaker condition than non-dominance as follows: for each

$\left(\sigma^{\prime}, t^{\prime}\right),\left(\sigma^{\prime \prime}, t^{\prime \prime}\right) \in f(C)$, each $c^{\prime}, c^{\prime \prime} \in C$ such that $\left(\sigma\left(c^{\prime}\right), t\left(c^{\prime}\right)\right)=\left(\sigma^{\prime}, t^{\prime}\right)$ and $\left(\sigma\left(c^{\prime \prime}\right), t\left(c^{\prime \prime}\right)\right)=\left(\sigma^{\prime \prime}, t^{\prime \prime}\right)$, and each $i \in I$, if there exists no $c^{\prime \prime \prime} \in C$ such that $L\left(\left(\sigma_{i}^{\prime}, t_{i}^{\prime}\right), c_{i}^{\prime}\right) \subseteq L\left(\left(\sigma_{i}^{\prime}, t_{i}^{\prime}\right), c_{i}^{\prime \prime \prime}\right)$ and $L\left(\left(\sigma_{i}^{\prime \prime}, t_{i}^{\prime \prime}\right), c_{i}^{\prime \prime}\right) \subseteq L\left(\left(\sigma_{i}^{\prime \prime}, t_{i}^{\prime \prime}\right), c_{i}^{\prime \prime \prime}\right)$, then there exists $c_{i} \in C_{i}$ such that $\left(\sigma_{i}^{\prime \prime}-\sigma_{i}^{\prime}\right) c_{i}=t_{i}^{\prime \prime}-t_{i}^{\prime}$, where

$$
\begin{aligned}
L\left(\left(\sigma_{i}, t_{i}\right), c_{i}\right) & \equiv\left\{\left(\sigma_{i}^{\prime}, t_{i}^{\prime}\right) \in I \times \mathbb{R} \mid-\left(\sigma_{i}-1\right) c_{i}+t_{i}\right. \\
& \left.\geq-\left(\sigma_{i}^{\prime}-1\right) c_{i}+t_{i}^{\prime}\right\}
\end{aligned}
$$

for each $i \in I$, each $c_{i} \in C_{i}$ and each $\left(\sigma_{i}, t_{i}\right) \in I \times \mathbb{R}$.

\section{Conclusion}

This paper studies secure implementability in queueing problems. Fujinaka and Wakayama [9] show a constancy result on secure implementation. Our model is a special case of their one and have many reasonable domains which do not satisfy minimal richness. However, we have the same constancy result under less restrictive domain-richness conditions. On the other hand, it remains to show domain-richness conditions for the existence of non-constant securely implementable social choice functions. However, our main result implies that it is difficult to find such conditions that are reasonable in the economic sense.

\section{Acknowledgements}

This paper is based on my M.A. thesis presented to the Graduate School of Economics, Osaka University. The author would like to thank an anonymous referee, Tatsuyoshi Saijo, Masaki Aoyagi, Yuji Fujinaka, Kazuhiko Hashimoto, Shuhei Morimoto, Shinji Ohseto, Shigehiro Serizawa, Takuma Wakayama, and seminar participants at the 2008 Japanese Economic Association Spring Meeting, Tohoku University, for their helpful comments.

\footnotetext{
${ }^{6}$ For the relationship among dual dominance, non-dominance, and secure implementability, see the supplementary note provided by Fujinaka and Wakayama [9] available online at: http://www.iser.osakau.ac.jp/library/dp/2007/DP0699N.pdf
}

The author is especially grateful to Tatsuyoshi Saijo, Yuji Fujinaka, and Takuma Wakayama for their valuable advices. The author acknowledges for the Global COE program of Osaka University for the financial supports. The responsibility for any errors that remain is entirely the author.

\section{REFERENCES}

[1] J. Suijs, "On Incentive Compatibility and Budget Balancedness in Public Decision Making," Economic Design, Vol. 2, No. 1, 1996, pp. 193-209. doi:10.1007/BF02499133

[2] T. Saijo, T. Sjöström and T. Yamato, "Secure Implementation," Theoretical Economics, Vol. 2, No. 3, 2007, pp. 203229.

[3] T. Cason, T. Saijo, T. Sjöström and T. Yamato, "Secure Implementation Experiments: Do Strategy-Proof Mechanisms Really Work?" Games and Economic Behavior, Vol. 57, No. 2, 2006, pp. 206-235. doi:10.1016/j.geb.2005.12.007

[4] D. Berga and B. Moreno, "Strategic Requirements with Indifference: Single-Peaked versus Single-Plateaued Preferences," Social Choice and Welfare, Vol. 32, No. 2, 2009, pp. 275-298. doi:10.1007/s00355-008-0323-y

[5] K. Nishizaki, "Secure Implementation in Discrete and Excludable Public Good Economies," Osaka Economic Papers, Vol. 61, No. 2, 2011, pp. 48-56.

[6] H. Mizukami and T. Wakayama, "Bossiness and Implementability in Pure Exchange Economies," Vol. 1461, 2005, pp. 126-140.

[7] K. Nishizaki, “An Equivalence of Secure Implementability and Full Implementability in Truthful Strategies in Pure Exchange Economies with Leontief Utility Functions," GCOE Discussion Paper Series No. 279, Osaka University, Osaka, 2012. http://www.iser.osaka-u.ac.jp/coe/dp/pdf/no.279_dp.pdf

[8] R. Kumar, "Secure Implementation in Production Economies," Department of Economics Working Paper Series No. 2011-02, Louisiana State University, Louisiana, 2009. http://bus.lsu.edu/McMillin/Working_Papers/pap11_02.pdf

[9] Y. Fujinaka and T. Wakayama, "Secure Implementation in Economies with Indivisible Objects and Money," Economics Letters, Vol. 100, No. 1, 2008, pp. 91-95. doi:10.1016/j.econlet.2007.11.009

[10] Y. Fujinaka and T. Wakayama, "Secure Implementation in Shapley-Scarf Housing Markets," Economic Theory, Vol. 48, No. 1, 2011, pp. 147-169. doi:10.1007/s00199-010-0538-x

[11] O. Bochet and T. Sakai, "Secure Implementation in Allotment Economies," Games and Economic Behavior, Vol. 68, No. 1, 2010, pp. 35-49. doi:10.1016/j.geb.2009.04.023

[12] Z. Ritz, "Restricted Domains, Arrow-Social Welfare Functions and Noncorruptible and Non-Manipulable Social Choice Correspondences: The Case of Private Alternatives," Mathematical Social Science, Vol. 4, No. 2, 1983, pp. $155-179$. 
[13] K. Nishizaki, "Secure Implementation in Queueing Problems," GCOE Discussion Paper Series No. 245, Osaka University, Osaka, 2012.

http://www.iser.osaka-u.ac.jp/coe/dp/pdf/no.245_dp_revised.pdf
[14] T. Saijo, "On Constant Maskin Monotonic Social Choice Functions," Journal of Economic Theory, Vol. 42, No. 2, 1987, pp. 382-386. doi:10.1016/0022-0531(87)90094-9 